Review

\title{
Hydrogen Sulfide Plays an Important Role by Influencing NLRP3 inflammasome
}

\author{
Honggang Wang ${ }^{1}$, Xingzhuo Shi², Mengyuan Qiu ${ }^{1}$, Shuangyu Lv¹, Hong Zheng ${ }^{1}$, Baohua Niu1 ${ }^{1}$, Huiyang \\ $\mathrm{Liu}^{1}$ \\ 1. Institute of Biomedical Informatics, Bioinformatics Center, School of Basic Medical Sciences, Henan University, Kaifeng, Henan, 475000, China \\ 2. School of Life Science, Henan University, Kaifeng, Henan, 475000, China \\ $\bowtie$ Corresponding author: Honggang Wang. E-mail: whg197167@vip.henu.edu.cn \\ (1) The author(s). This is an open access article distributed under the terms of the Creative Commons Attribution License (https://creativecommons.org/licenses/by/4.0/). \\ See http://ivyspring.com/terms for full terms and conditions.
}

Received: 2020.04.29; Accepted: 2020.08.12; Published: 2020.08.25

\begin{abstract}
Inflammasome is a complex composed of several proteins and an important part of the natural immune system. Nucleotide-binding oligomerization domain-like receptor protein 3 (NLRP3) inflammasome is composed of NLRP3, apoptosis associated speck like protein (ASC) and pro-caspase-1. It plays an important role in many diseases. Hydrogen sulfide $\left(\mathrm{H}_{2} \mathrm{~S}\right)$ is an important signaling molecule that regulates many physiological and pathological processes. Recent studies indicated that $\mathrm{H}_{2} \mathrm{~S}$ played anti-inflammatory and pro-inflammatory roles in many diseases through influencing NLRP3 inflammasome, but its mechanism was not fully understood. This article reviewed the progress about the effects of $\mathrm{H}_{2} \mathrm{~S}$ on NLRP3 inflammasome and its mechanisms involved in recent years to provide theoretical basis for in-depth study.
\end{abstract}

Key words: Hydrogen sulfide; NLRP3 inflammasome; renal protection; neuroprotection; diabetes

\section{Introduction}

A decade ago, inflammasome was described as a large intracellular signaling platform, which contains a cytoplasmic pattern recognition receptor, especially a nucleotide-binding oligomerization domain-like receptor (NLR). Although several types of inflammasomes have been identified so far, nucleotide-binding oligomerization domain-like receptor protein 3 (NLRP3) inflammasome is the most characteristic one [1]. The researches indicated that the abnormal activation of NLRP3 inflammasome was related to the pathogenesis of various autoimmune, chronic inflammatory and metabolic diseases, including gout, atherosclerosis and type 2 diabetes [2-4].

Hydrogen sulfide $\left(\mathrm{H}_{2} \mathrm{~S}\right)$ is an important signaling molecule that regulates many physiological and pathological processes. Recent studies indicated that $\mathrm{H}_{2} \mathrm{~S}$ played anti-inflammatory and pro-inflammatory roles in many diseases through influencing NLRP3 inflammasome, but its mechanism was not fully understood. In this review, we summarized the recent studies on the anti-inflammatory or pro-inflammatory effects of $\mathrm{H}_{2} \mathrm{~S}$ on NLRP3 inflammasome in a variety of diseases to provide ideas for the relevant basic research in the future.

\section{Overview of NLRP3 inflammasome}

Inflammasome is a complex composed of several proteins and an important part of the natural immune system. A variety of inflammasome have been found: NLRP1, NLRP2, NLRP3, NLRP6, NLRP7, NLRP12, NLRC4, IPAF and AIM2. NLRP3 inflammasome is the most thoroughly studied one, which is composed of NLRP3, apoptosis associated speck like protein (ASC) and caspase-1 precursor (pro-caspase-1) (Figure 1) [5-10]. By activating caspase-1, NLRP3 inflammasome can induce the maturation and secretion of pro-inflammatory factors: Interleukin-1beta (IL-1 $\beta$ ) and Interleukin-18 (IL-18). Mature IL-1 $\beta$ is an effective proinflammatory mediator in many immune responses, including the recruitment of natural immune cells to the infection site and the regulation of 
adaptive immune cells. Mature IL-18 plays an important role in the production of IFN- $\gamma$ and the enhancing the cytolytic activity of natural killer cells and T cells [11]. The actived caspase- 1 also induces a proinflammatory form of cell death [12]. Therefore, NLRP3 inflammasome can regulate the immune response of the body and strictly control the inflammatory reaction in the pathophysiological process. NLRP3 inflammasome can be activated by different stimuli including pathogen associated molecular patterns (PAMPs) and damage associated molecular pattern (DAMPs). The first stimuli, which is mediated by proinflammatory pathways, such as toll like receptor (TLR)-mediated activation of nuclear factor-kB (NF-kB), promotes the upregulation of the components of the inflammasome. The second stimuli, including reactive oxygen species (ROS) production, intracellular potassium $\left(\mathrm{K}^{+}\right)$ concentration and the distuption of lysosomal membrane, promotes the assembly of inflammasome and leads to the activation of caspase- 1 , which can catalyze the pro-IL-1 $\beta$ into active IL-1 $\beta[1,9,13-15]$. NLRP3 inflammasome has been reported to be related to the pathogenesis of many complex diseases, such as type 2 diabetes [16], atherosclerosis [17-20], obesity and gout [21], Alzheimer's disease and Parkinson's disease[22, 23].

\section{Overview of $\mathrm{H}_{2} \mathrm{~S}$ and the mechanism of $\mathrm{H}_{2} \mathrm{~S}$ acting on NLRP3 inflammasome}

Over the years, $\mathrm{H}_{2} \mathrm{~S}$ has been regarded as a toxic gas with an unpleasant smell. However, since the 1990s, more and more researches have indicated that

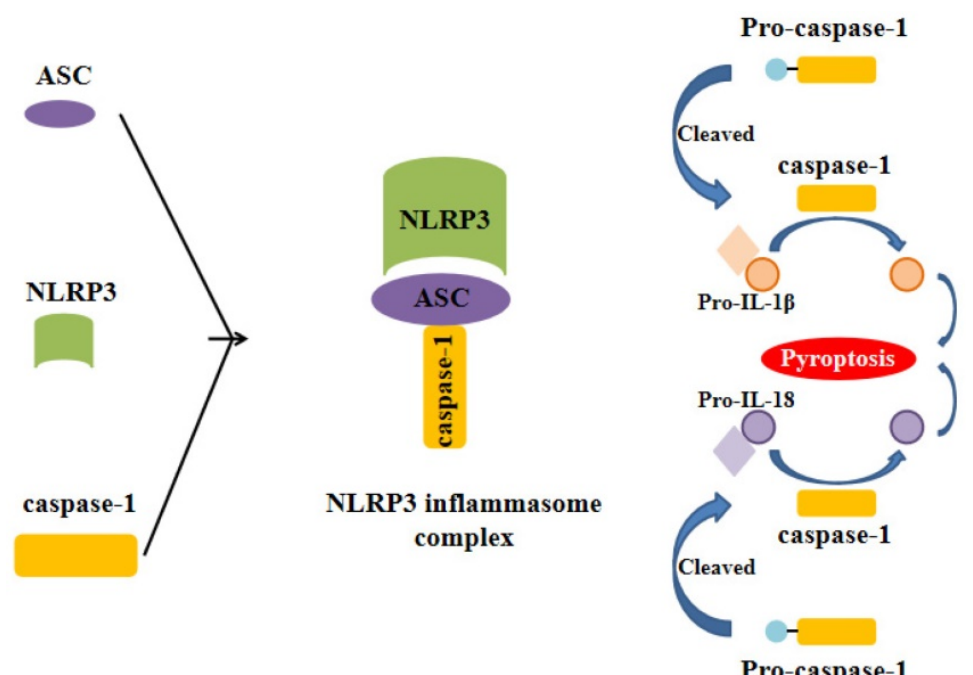

Figure 1. Formation of the NLRP3 inflammasome. The activation of NLRP3 inflammasome involves the assembling of the components of NLRP3 inflammasome (NLRP3, ASC and caspase-1) to form a complete NLRP3 inflammasome complex. This inflammasome complex allows the cleavage of pre-caspase- 1 into its active isomer, caspase-1, which then cleaves pro-IL-1 $\beta$ and pro-IL-18 to their active isomers IL-1 $\beta$ and IL-18 respectively. The increase of these pro-inflammatory proteins eventually leads to pyroptosis.
$\mathrm{H}_{2} \mathrm{~S}$, together with nitric oxide (NO) and carbon monoxide (CO), belongs to a class of gasotransmitters.There is increasing evidence that $\mathrm{H}_{2} \mathrm{~S}$ can be produced in multiple organ systems in mammals, including humans and fish [24-27]. In mammalian cells, $\mathrm{H}_{2} \mathrm{~S}$ is produced by endogenous enzymatic and non-enzymatic pathways. The enzymatic generation of $\mathrm{H}_{2} \mathrm{~S}$, which may be important for the regulation in given cells under special conditions, is the focus of the research. Several different mammalian enzymatic systems for $\mathrm{H}_{2} \mathrm{~S}$ production have been described in detail. Most commonly, three typical $\mathrm{H}_{2} \mathrm{~S}$-producing enzymes are identified: cystathionine-gamma-lyase (CSE), cystathionine-beta-synthase (CBS) and 3-mercaptopyruvate thiotransferase (3-MST). Cystathionine is produced by $\beta$-substitution reaction of homocysteine with serine catalyzed by CBS. CSE catalyzes the elimination of $\alpha, \gamma$-cysteine of cystathionine to produce cystenine. Under the catalysis of CBS and CSE, cysteine can form $\mathrm{H}_{2} \mathrm{~S}$ through $\beta$ elimination reaction. 3-mercaptopyruvate (3-MP) is produced by transferring amines from cystine to a-ketoglutarate via cysteine aminotransferase (CAT). 3-MST catalyzes the sulphur of 3-MP to convert into $\mathrm{H}_{2} \mathrm{~S}$ (Figure 2) [28]. For the inhibition of the synthesis of endogenous $\mathrm{H}_{2} \mathrm{~S}$, there are several small molecular compounds, which can inhibit the synthesis of endogenous $\mathrm{H}_{2} \mathrm{~S}$, targeting at three kinds of $\mathrm{H}_{2} \mathrm{~S}$ producing enzymes. Although these compounds have their limitations (potency, selectivity), these molecules, especially in combination with genetic methods, can be used to describe biological processes involving endogenous $\mathrm{H}_{2} \mathrm{~S}$ production [29]. $\mathrm{H}_{2} \mathrm{~S}$ has the physiological functions of relaxing blood vessels, lowering blood pressure [30, 31], antiapoptosis [32], anti-inflammation [33], anti-oxidation and regulating endoplasmic reticulum stress [34]. At present, the effect of $\mathrm{H}_{2} \mathrm{~S}$ on NLRP3 inflammasome has gradually become a research hotspot.

$\mathrm{H}_{2} \mathrm{~S}$ can inhibit TLR4/NF- $\kappa$ B pathway [35, 36], clear ROS [37], suppress $\mathrm{K}^{+}$efflux [38] and promote lysosomal membrane rupture [39], which are related to NLRP3 activation. Therefore, it can be inferred that $\mathrm{H}_{2} \mathrm{~S}$ can act on NLRP3 inflammasome through the above pathways (Figure 3).

\section{$\mathrm{H}_{2} \mathrm{~S}$ plays liver protection roles by influencing NLRP3 inflammasome}

Exogenous $\mathrm{H}_{2} \mathrm{~S}$ can inhibit the inflammatory response of hepatocytes by influencing NLRP3 inflammasome to protect 
liver. Our previous studies showed that in the lipopolysaccharide (LPS)-induced hepatocyte inflammation model, the protein expression level of NLRP3 inflammasome and the level of IL-1 $\beta$ were significantly increased and $\mathrm{H}_{2} \mathrm{~S}$ reversed these changes, which indicated that $\mathrm{H}_{2} \mathrm{~S}$ could significantly inhibit NLRP3 inflammasome-mediated inflammatory response [40]. We also found that in the oleic acid (OA)-induced hepatocyte inflammation model, the level of autophagy was decreased significantly and the protein expression level of NLRP3 inflammasome was increased, while exogenous $\mathrm{H}_{2} \mathrm{~S}$ could counteract the OA-induced change. 3-MA, an autophagy inhibitor, could reverse the inhibitory effect of $\mathrm{H}_{2} \mathrm{~S}$ on NLRP3 inflammasome induced by $\mathrm{OA}$, indicating that exogenous $\mathrm{H}_{2} \mathrm{~S}$ could inhibit the protein expression of NLRP3 inflammasome by promoting autophagy in OA-induced hepatocyte. Our in-depth mechanism research showed that in OA-induced hepatocyte, $\mathrm{H}_{2} \mathrm{~S}$ could inhibit the NLRP3 inflammasome-mediated inflammation and activate the AMP-activated protein kinase (AMPK)/ mammalian target of rapamycin (mTOR) pathway and autophagy. 3-MA, an autophagy inhibitor, could counteract the effect of $\mathrm{H}_{2} \mathrm{~S}$, suggesting that autophagy mediated the effect of $\mathrm{H}_{2} \mathrm{~S}$ on NLRP3 inflammasome-mediated inflammation. In addition, compound C, an AMPK inhibitor, could inhibit autophagy and counteract the anti-inflammatory effect of exogenous $\mathrm{H}_{2} \mathrm{~S}$. In summary, exogenous $\mathrm{H}_{2} \mathrm{~S}$ inhibited NLRP3 inflammasome-mediated inflammation of hepatocytes through promoting autophagy via AMPK/mTOR pathway (Figure 4) [41, 42]. Through consulting a large number of related literatures, we found that exogenous $\mathrm{H}_{2} \mathrm{~S}$ could inhibit endoplasmic reticulum stress (ERS) in many diseases [28], and there was interaction between ERS and NLRP3 inflammasome [43], so whether exogenous
$\mathrm{H}_{2} \mathrm{~S}$ can inhibit NLRP3 inflammasome-mediated inflammatory response through ERS needs further study. One of the liver injuries in nonalcoholic fatty liver disease (NAFLD) is inflammatory liver injury [44], in view of our previous studies, it can be inferred that exogenous $\mathrm{H}_{2} \mathrm{~S}$ can atteuate NAFLD by inhibiting NLRP3 inflammasome, which is still further proven. NLRP3 inflammasome will be an important target of NAFLD treatment.

Paraquat (PQ) poisoning is a serious clinical problem due to the lack of specific antidotes and the accidental or suicide PQ intake leading to high mortality. Studies have shown that oxidative stress and ROS-mediated inflammation were the main causes of PQ poisoning [45]. Liver is the main source of endogenous antioxidants and plays an important role in enzyme metabolism and detoxification. Therefore, the liver is more vulnerable to $P Q$ poisoning [46, 47]. It has been reported that PQ activated NLRP3 inflammasome, resulting in the secretion of IL-1 $\beta$ and IL-18 in macrophages. Therefore, the inhibition of NLRP3 inflammasome-mediated inflammatory response may be beneficial to the treatment of PQ poisoning [48, 49]. Zhenning Liu et al.found that in PQ-induced rat liver injury, $\mathrm{H}_{2} \mathrm{~S}$ could significantly inhibit the protein expression level of NLRP3 inflammasome, pro-caspase- 1 and the secretion of IL-1 $\beta$ and activate Nrf2 signal pathway. The nuclear factor erythroid-2-related factor 2 (Nrf2) gene knockout or siRNA-Nrf2 could counteract the protective effect of $\mathrm{H}_{2} \mathrm{~S}$, suggesting that $\mathrm{H}_{2} \mathrm{~S}$ could alleviate PQ-induced liver injury by inhibiting NLRP3 inflammasome-mediated inflammatory response through Nrf2 signal pathway (Figure 4)[50]. The inhibitory effect of $\mathrm{H}_{2} \mathrm{~S}$ on NLRP3 inflammasome has therapeutic effect on PQ-induced liver injury.

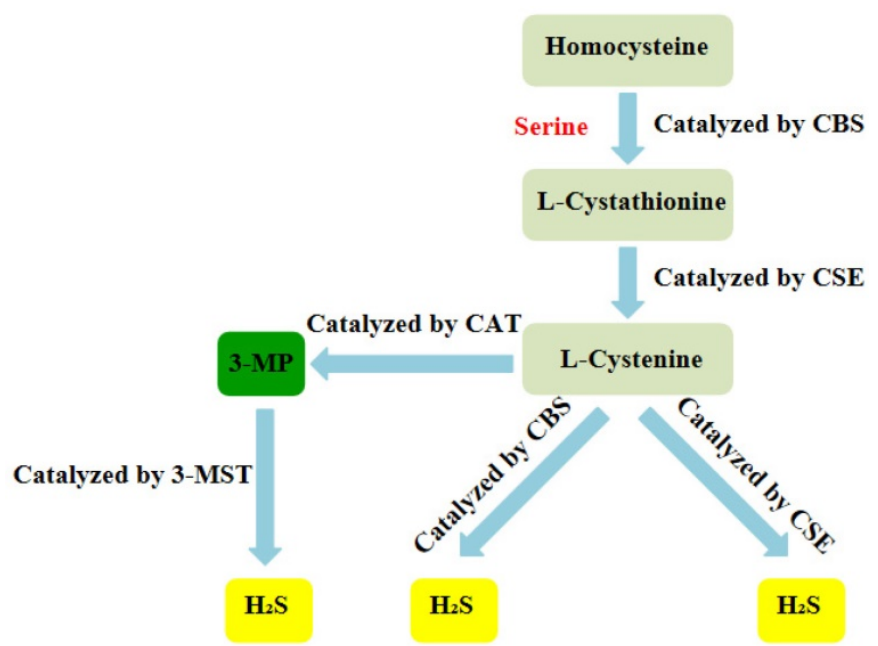

Figure 2. Summary of the production process of endogenous $\mathrm{H}_{2} \mathrm{~S}$. CBS:cystathionine-beta-synthase; CSE:cystathionine-gamma-lyase; 3-MST: 3-mercaptopyruvate thiotransferase; 3-MP:3-mercaptopyruvate;CAT:cysteine aminotransferase 


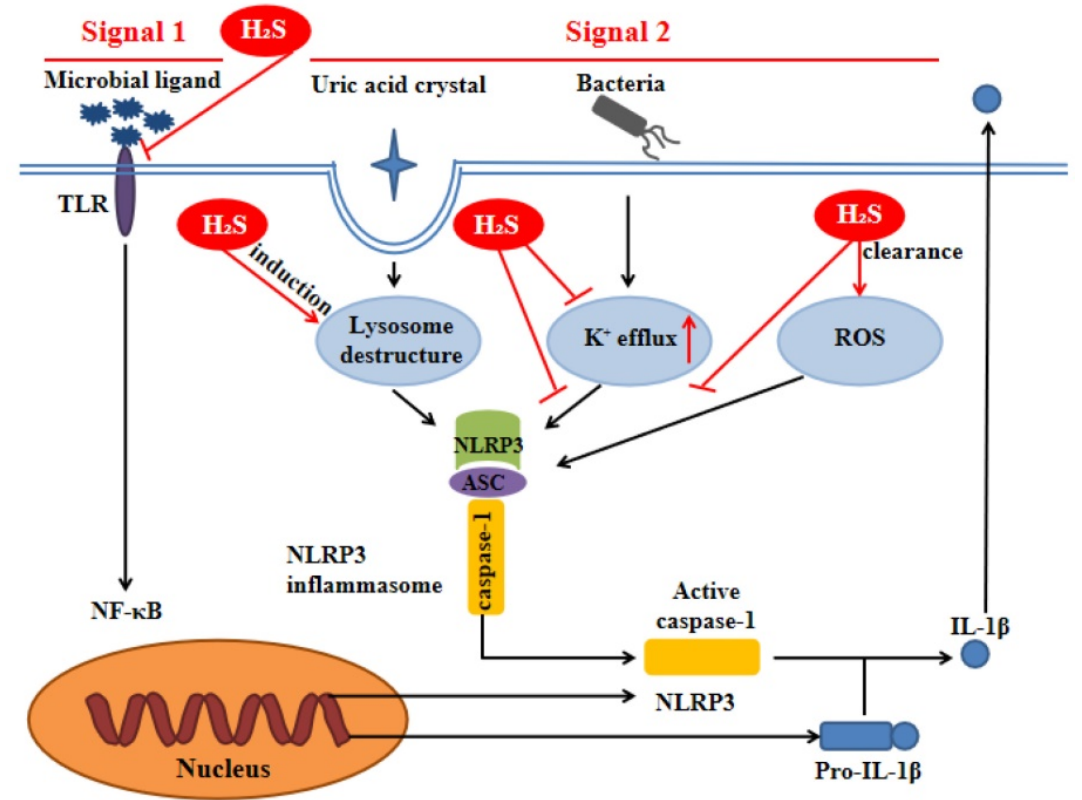

Figure 3. $\mathrm{H}_{2} \mathrm{~S}$ influences NLRP3 inflammasome through signal $\mathbf{I}$ and signal 2 . Signal 1 is mediated by microbial ligands recognized by TLR which activates the NF-kB pathway to promote the protein expression level of pro-IL-1 $\beta$ and NLRP3. The signal 2 promotes the assembly of the NLRP3 inflammasome complex. Under noninfectious conditions, $\mathrm{K}^{+}$efflux leads to the activation of NLRP3 inflammasome. Various endogenous and exogenous particulates, including uric acid crystal, promote lysosomal damage to activate NLRP3 inflammasome. Additionally, the increase of ROS level in the cell also activates the NLRP3 inflammasome. $\mathrm{H}_{2} \mathrm{~S}$ can influence NLRP3 inflammasome through the above pathways. ASC: apoptosis-associated speck-like protein containing a C-terminal caspase recruitment domain; NF-kB: nuclear factor kappa-light-chain-enhancer of activated B cells; ROS: reactive oxygen species; TLR:toll-like receptor

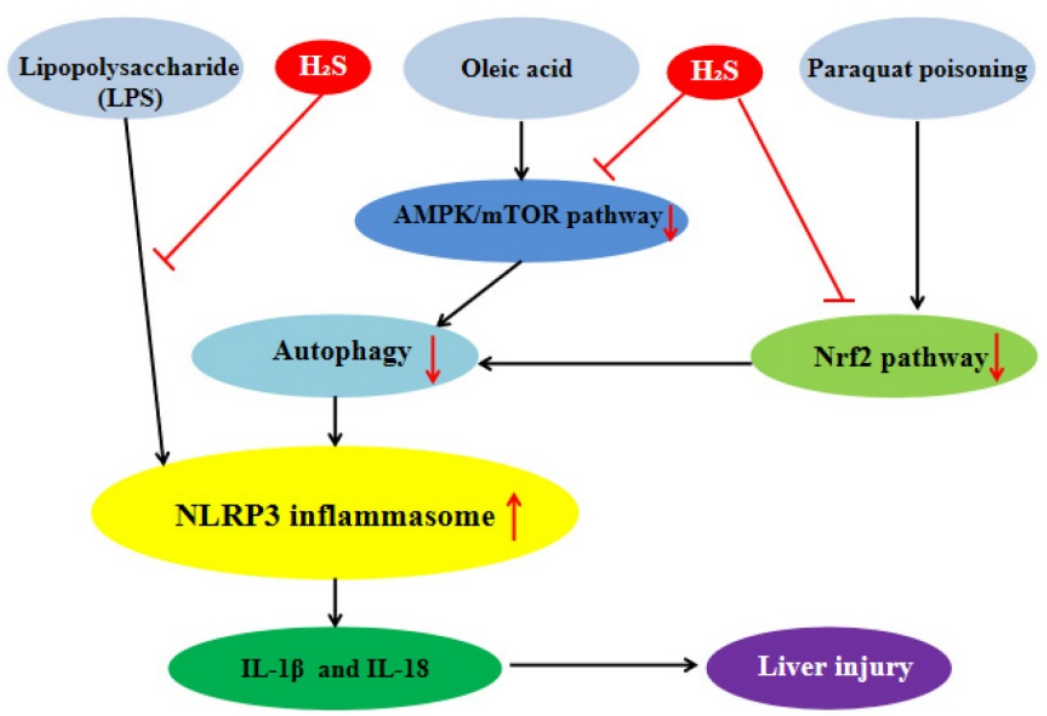

Figure 4. $\mathrm{H}_{2} \mathrm{~S}$ plays liver protection roles by influencing NLRP3 inflammasome. $\mathrm{H}_{2} \mathrm{~S}$ significantly inhibits NLRP3 inflammasome-mediated inflammatory injury induced by lipopolysaccharide and suppress NLRP3 inflammasome-mediated inflammatory injury induced by oleric acid through promoting autophagy via AMPK/mTOR pathway. $\mathrm{H}_{2} \mathrm{~S}$ can alleviate NLRP3 inflammasome-mediated inflammatory injury induced by paraquat poisoning through Nrf2 signal pathway. AMPK: AMP-activated protein kinase; mTOR:mammalian target of rapamycin; Nrf2:nuclear factor erythroid-2-related factor 2

\section{$\mathrm{H}_{2} \mathrm{~S}$ plays renal protection role by influencing NLRP3 inflammasome}

Acute renal injury is a clinical syndrome caused by many factors, which is characterized by rapid decline of renal function [51]. It has been reported that NLRP3 inflammasome participated in the inflammatory process, which might be the key to the development of acute renal injury [52]. Yuhong Chen, et al. found that exogenous $\mathrm{H}_{2} \mathrm{~S}$ could inhibit the protein expression level of NLRP3 inflammasome to attenuate LPS-induced rat acute renal injury [53]. The signal transduction mechanism of the above-mentioned action of $\mathrm{H}_{2} \mathrm{~S}$ needed further study. Renal fibrosis and renal injury are important clinical features of many chronic kidney diseases (CKDS) [54]. It has been shown that NLRP3 inflammasome was involved in the pathogenesis of CKDS $[55,56]$. In the model of injury and fibrosis of unilateral ureteral obstruction (UUO) mice, exogenous $\mathrm{H}_{2} \mathrm{~S}$ could 
alleviate macrophage infiltration, tissue fibrosis, and inhibit NF-KB and IL-4/signal transducer and activator of transcription 6 (STAT6) signaling pathways and NLRP3 inflammasome, and NLRP3 inhibitor had the effect similar to that of $\mathrm{H}_{2} \mathrm{~S}$, which suggested that $\mathrm{H}_{2} \mathrm{~S}$ alleviated renal fibrosis via inhibiting NLRP3 inflammasome. These studies also showed that NLRP3 inflammasome activation contributes to macrophage infiltration and tissue fibrosis, and NF-kB and IL-4/STAT6 signaling pathways were related to macrophage infiltration. So, it could be inferred that $\mathrm{H}_{2} \mathrm{~S}$ alleviates renal fibrosis in response to UUO by suppressing macrophage infiltration through inhibition of NLRP3 inflammasome via NF-KB and IL-4/STAT6 signaling pathways, which needed to be further proven [57-59].

\section{$\mathrm{H}_{2} \mathrm{~S}$ plays neuroprotection role by influencing NLRP3 inflammasome}

Intracerebral haemorrhage $(\mathrm{ICH})$ is a devastating stroke with high mortality and incidence rate. Countless evidences from preclinical and clinical studies suggested that inflammatory mechanisms were involved in ICH-induced secondary brain injury $[60,61]$. Studies have shown that the activation of NLRP3 inflammasome played an important role in the development of neuroinflammation after ICH [62]. Exogenous $\mathrm{H}_{2} \mathrm{~S}$ could inhibit the activation of NLRP3 inflammasome and the subsequent release of IL-1 $\beta$ induced by ICH. Purinergic P2X7 receptor (P2X7R) is an ATP gated, non-selective cation channel, belonging to the family of ionotropic P2X receptors. It was reported that P2X7R interacts with NLRP3 inflammasome, which was responsible for the recruitment and activation of NLRP3. $\mathrm{H}_{2} \mathrm{~S}$ could suppress the P2X7R expression and the overexpression of $\mathrm{P} 2 \mathrm{X} 7 \mathrm{R}$ could upregulate the expression of NLRP3 inflammasome on microglia after ICH. These results suggested that $\mathrm{H}_{2} \mathrm{~S}$ could suppress NLRP3 inflammasome-mediated neuroinflammation by inhibiting P2X7 receptor after $\mathrm{ICH}$ in rats [63]. As we all know, $\mathrm{H}_{2} \mathrm{~S}$ plays an important role in antioxidation, therefore, it is reasonable to speculate that in addition to inhibiting the expression of P2X7R, $\mathrm{H}_{2} \mathrm{~S}$ can also inhibit NLRP3 inflammasome-mediated neuroinflammation by directly eliminating the ROS after ICH, which still needs to be studied. Ischemic stroke is one of the main causes of the disability and death worldwide [64]. Inflammatory response was often involved in ischemic stroke injury [65]. $\mathrm{H}_{2} \mathrm{~S}$ could play a neuroprotective role by inhibiting the activation of NLRP3 inflammasome in ischemic brain [66]. The inhibitory effect of $\mathrm{H}_{2} \mathrm{~S}$ on NLRP3 inflammasome has potential therapeutic value for ischemic stroke injury.

\section{$\mathrm{H}_{2} \mathrm{~S}$ inhibits NLRP3 inflammasome in macrophages}

Fatty acids (FA) have been shown to induce inflammation in primary human macrophages [67]. In FA-induced RAW264.7 cell, the protein expression level of NLRP3 inflammasome and the level of IL-1 $\beta$ and IL-18 were increased and the the TLR4/ NF-KB pathway was activated, while $\mathrm{H}_{2} \mathrm{~S}$ could counteract these changes. NLRP3 siRNA reduced the level of IL-1 $\beta$ and IL-18 induced by FA, suggesting that NLRP3 inflammasome mediated FA-induced inflammation. TLR4 inhibitor and NF-KB inhibitor reduced the protein expression level of NLRP3 inflammasome induced by FA, suggesting that TLR4/NF-kB mediated the activation of NLRP3 inflammasome induced by FA. In summary, it could be inferred that exogenous $\mathrm{H}_{2} \mathrm{~S}$ suppressed NLRP3 inflammasome-mediated inflammation by inhibiting TLR4/NF-kB pathway in FA-induced RAW264.7 cells, which needed further study [35]. It has been shown that mitochondrial uncoupling protein 2 (UCP2) regulated NLRP3 inflammasome by inducing lipid synthesis in macrophages [68], So whether exogenous $\mathrm{H}_{2} \mathrm{~S}$ could regulate lipid synthesis pathway by inhibiting NLRP3 inflammasome needed further research. The activation of NLRP3 inflammasome in macrophages has been considered to be involved in diseases [69-75]. Exogenous $\mathrm{H}_{2} \mathrm{~S}$ could inhibit NLRP3 inflammasome-mediated inflammation in human macrophages exposed to $\mathrm{H}_{2} \mathrm{O}_{2}$, which was related to the reduction of mitochondrion ROS (mtROS). The in depth study on the mechanism of the above effect showed that $\mathrm{H}_{2} \mathrm{~S}$ decreased the production of mtROS by S-sulfhydrating c-Jun at cysteine-269. The suppression of S-sulfhydrated c-Jun of $\mathrm{H}_{2} \mathrm{~S}$ could reverse the inhibition of $\mathrm{H}_{2} \mathrm{~S}$ on NLRP3 inflammasome, suggesting that $\mathrm{H}_{2} \mathrm{~S}$ inhibited the NLRP3 inflflammasome activation via sulfhydration of c-Jun at cysteine-269. The S-sulfhydrated c-Jun of $\mathrm{H}_{2} \mathrm{~S}$ increased SIRT3 expression, and in the macrophages of SIRT3-/- mice exposed to $\mathrm{H}_{2} \mathrm{O}_{2}$, the inhibition of $\mathrm{H}_{2} \mathrm{~S}$ on NLRP3 inflammasome was diminished, which suggested that $\mathrm{H}_{2} \mathrm{~S}$ inhibited NLRP3 inflammasome through SIRT3 [76]. The modification of c-jun by $\mathrm{H}_{2} \mathrm{~S}$ may provide ideas for the treatment of NLRP3 inflammasome involved diseases. In primary human macrophages, $\mathrm{H}_{2} \mathrm{~S}$ inhibited monosodium urate (MSU)-induced NLRP3 inflammasome activation, xanthine oxidase $(\mathrm{XO})$ activity and $\mathrm{mtROS}$ generation while febuxostat (a XO-inhibitor) diminished MSU-induced mtROS generation and NLRP3 inflammasome activation, which suggested $\mathrm{H}_{2} \mathrm{~S}$ was capable of inhibiting NLRP3 inflammasome by 
suppressing XO activity [77, 78].

\section{$\mathrm{H}_{2} \mathrm{~S}$ plays a protective role by influencing NLRP3 inflammasome in diabetes}

Chronic, low-level systemic and aseptic inflammation is a common feature of diabetic cardiomyopathy (DCM) [79]. A study showed that inhibiting NLRP3 inflammasome could significantly alleviate DCM [80]. $\mathrm{H}_{2} \mathrm{~S}$ has been reported to protect cardiomyocytes from inflammation and cell death in diabetic models [81, 82]. In high glucose(HG)-induced H9c2 cardiac cells, the protein expression level of NLRP3 inflammasome and the level of IL-1 $\beta$ and IL-18 were increased and the the TLR4/NF-kB pathway was activated, while $\mathrm{H}_{2} \mathrm{~S}$ could counteract these changes. NLRP3 siRNA reduced the level of HG-induced IL- $1 \beta$ and IL-18, indicating that NLRP3 inflammasome mediated HG-induced inflammation. TLR4 inhibitor and NF-KB inhibitor reduced the protein expression level of HG-induced NLRP3 inflammasome, indicating that TLR4/NF-KB mediated the activation of NLRP3 inflammasome in HG-induced H9c2 cardiac cells. In summary, it could be inferred that exogenous $\mathrm{H}_{2} \mathrm{~S}$ suppressed NLRP3 inflammasome-mediated inflammation by inhibiting TLR4/NF-kB pathway in H9c2 cardiac cells, which needed further study [36]. HG could cause lipid metabolism disorder, and NLRP3 inflammasome participated in lipid metabolism process [70], so whether $\mathrm{H}_{2} \mathrm{~S}$ could improve lipid metabolism through NLRP3 inflammasome to alleviate DCM needed further study. Diabetic retinopathy is a common complication of diabetes mellitus, which is also the main cause of visual impairment and blindness [83]. Chronic hyperglycemia damaged not only the retinal vessels but also the retinal pigment epithelial cells (RPE)[84]. In HG-induced RPE cells, HG increased the production of intracellular ROS and the level of IL-1 $\beta$ and IL-18 and activated NLRP3 inflammasome while $\mathrm{H}_{2} \mathrm{~S}$ counteracted these changes. Knock down of NLRP3 decreased the level of IL-1 $\beta$ and IL-18, suggesting that NLRP3 inflammasome mediated the HG-induced inflammation. In conclusion, $\mathrm{H}_{2} \mathrm{~S}$ inhibits HG-induced inflammation of human retinal pigment epithelial cells through inhibiting NLRP3 inflammasome [85]. In HG-induced 3T3-L1 adipocytes, $\mathrm{H}_{2} \mathrm{~S}$ has the effect similar to the above [86]. $\mathrm{H}_{2} \mathrm{~S}$ has therapeutic effect on diabetes through inhibiting NLRP3 inflammasome. Diabetesaccelerated atherosclerosis is the most common cardiovascular complication of diabetes mellitus [87]. $\mathrm{H}_{2} \mathrm{~S}$ also decreased the HG-induced endothelial injury and the protein expression level of NLRP3 inflammasome in vivo and in vitro, while the silencing of NLRP3 had the effect similar to that of
$\mathrm{H}_{2} \mathrm{~S}$, suggesting that $\mathrm{H}_{2} \mathrm{~S}$ protected against diabetes-accelerated atherosclerosis by inhibiting the activation of NLRP3 inflammasome [88]. It provided the new evidences for the treatment of cardiovascular diseases with $\mathrm{H}_{2} \mathrm{~S}$. NLRP3 inflammasome is related to lipid metabolism, and $\mathrm{H}_{2} \mathrm{~S}$ can promote lipolysis [89], so It can be deduced that $\mathrm{H}_{2} \mathrm{~S}$ can promote lipolysis by inhibiting NLRP3 inflammasome against diabetes-accelerated atherosclerosis, which needs further study.

\section{$\mathrm{H}_{2} \mathrm{~S}$ plays a protective role in other inflammatory reactions by inhibiting NLRP3 inflammasome}

Repeated exposure of mice to high concentrations of ozone has been shown to cause chronic lung inflammation, emphysema and airflow restriction [90]. In ozone exposed mice, ozone increased the protein expression level of the NLRP3 inflammasome, cleavage caspase- 1 and the level of p38 mitogen-activated protein kinases (MAPK) phosphorylation and decreased the level of protein kinase B (Akt) phosphorylation, while $\mathrm{H}_{2} \mathrm{~S}$ counteracted these changes [91]. Therefore, it can be inferred that $\mathrm{H}_{2} \mathrm{~S}$ can alleviate lung inflammation caused by ozone exposure through supressing NLRP3 inflammasome and p38MAPK/Akt pathways, which needs to be proven by using specific inhibitors or specific knock-out mice to block certain pathways. It is reported that NLRP3 inflammasome mediated dextran sodium sulfate (DSS)-induced colitis. $\mathrm{H}_{2} \mathrm{~S}$ could reduce the inflammation of colitis induced by DSS through inhibiting the activation of NF- $\mathrm{kB}$ pathway, so it could be infered that $\mathrm{H}_{2} \mathrm{~S}$ could relieve DDS-induced colitis through suppressing NLRP3 inflammasome via NF- $\mathrm{\kappa B}$ pathway, which neede further study [92-95]. In DSS-induced colitis, $\mathrm{H}_{2} \mathrm{~S}$ decreased the protein expression level of NLRP3 inflammasome, pro-caspase- 1 , and $\mathrm{Nrf} 2$ and the silencing Nrf2 has the effects similar to the above, which indicated that $\mathrm{H}_{2} \mathrm{~S}$ inhibited NLRP3 inflammasome through $\mathrm{Nrf2}$ pathway [96]. $\mathrm{H}_{2} \mathrm{~S}$ could ameliorate endothelial dysfunction and hypertension $[97,98]$. The mechanism research showed that $\mathrm{H}_{2} \mathrm{~S}$ could improve endothelium-dependent contraction and relaxation and reduce the protein expression levels of NLRP3 inflammasome and the level of IL-1 $\beta$ in spontaneously hypertensive rats. The above ameliorative effects of $\mathrm{H}_{2} \mathrm{~S}$ were abolished by LPS (a NLRP3 activator), suggesting that $\mathrm{H}_{2} \mathrm{~S}$ ameliorated endothelial dysfunction and hypertension by inhibiting NLRP3 inflammasome [99]. It suggested that the effect of $\mathrm{H}_{2} \mathrm{~S}$ on NLRP3 has potential therapeutic function in the treatment of hypertension. 


\section{$\mathrm{H}_{2} \mathrm{~S}$ promotes NLRP3 inflammasome to promote diseases development}

Besides the anti-inflammatory effect, $\mathrm{H}_{2} \mathrm{~S}$ can also promote the inflammatory reaction to participate in the development of diseases through promoting NLRP3 inflammasome. The studies showed that in human monocyte, $\mathrm{H}_{2} \mathrm{~S}$ could induce NLRP3 inflammasome dependent secretion of IL-1 $\beta$ and IL-18 by promoting the assembly of NLRP3 inflammasome to contribute to diseases development [100, 101]. In broiler thymus, the atmospheric $\mathrm{H}_{2} \mathrm{~S}$ could activate NLRP3 inflammasome to decrease thymus index, thymus immunoglobulin and $\mathrm{T}$ lymphocyte number and damaged thymus morphology, which suggested that the atmospheric $\mathrm{H}_{2} \mathrm{~S}$ has immunotoxicity. The mechanism study of the above actions showed that TLR-7/myeloid differentiation factor 88(MyD88)/NF$\kappa B$ pathway was activated by $\mathrm{H}_{2} \mathrm{~S}$. So It can be inferred that $\mathrm{H}_{2} \mathrm{~S}$ might activate NLRP3 inflammasome via TLR-7/MyD88/NF-kB pathway, which needed to be further proven [102].Under what conditions does $\mathrm{H}_{2} \mathrm{~S}$ promote NLRP3 needs further study.

\section{Summary}

$\mathrm{H}_{2} \mathrm{~S}$ has both anti-inflammatory and pro-inflammatory effects and the mechanism has not been fully studied. The current researches have showed that the mechanism of $\mathrm{H}_{2} \mathrm{~S}$ in inflammation was related to the concentration of $\mathrm{H}_{2} \mathrm{~S}$, the stage of development of inflammatory diseases and the types of tissues affected by $\mathrm{H}_{2} \mathrm{~S}$. For example, the low concentration of $\mathrm{H}_{2} \mathrm{~S}$ can inhibit the inflammatory response to reduce the inflammatory damage of tissues and organs, while the high concentration of $\mathrm{H}_{2} \mathrm{~S}$ can promote the inflammatory response to aggravate the inflammatory damage. Similar to the above, the effects of $\mathrm{H}_{2} \mathrm{~S}$ on NLRP3 inflammasome are either inhibition or promotion. Whether $\mathrm{H}_{2} \mathrm{~S}$ can inhibit NLRP3 inflammasome to play a protective role or promote NLRP3 inflammasome to participate in the development of diseases, especially the latter, needs further study. No matter what role $\mathrm{H}_{2} \mathrm{~S}$ plays, the research and development of $\mathrm{H}_{2} \mathrm{~S}$ donor or $\mathrm{H}_{2} \mathrm{~S}$ inhibitor related drugs will provide a new way for the treatment of inflammatory diseases. In addition, the mechanism of $\mathrm{H}_{2} \mathrm{~S}$ acting on NLRP3inflammasome has not been fully studied. For example, whether $\mathrm{H}_{2} \mathrm{~S}$ can act on NLRP3 inflammasome by influencing lysosomal rupture or $\mathrm{K}^{+}$efflux remains to be elucidated.

In conclusion, NLRP3 inflammasome may be a potential target for $\mathrm{H}_{2} \mathrm{~S}$ therapy in inflammatory diseases with the in-depth study of the effect of $\mathrm{H}_{2} \mathrm{~S}$ on NLRP3 inflammasome.

\section{Acknowledgments}

This work was supported by grants from key scientific and technological projects in Henan Province, China (Grant number 202102310153).

\section{Competing Interests}

The authors have declared that no competing interest exists.

\section{References}

1. Jo EK, Kim JK, Shin DM, Sasakawa C. Molecular mechanisms regulating NLRP3 inflammasome activation. Cell Mol Immunol. 2016; 13: 148-59.

2. Ozaki E, Campbell M, Doyle SL. Targeting the NLRP3 inflammasome in chronic inflammatory diseases: current perspectives. J Inflamm Res. 2015; 8: 15-27.

3. Menu P, Vince JE. The NLRP3 inflammasome in health and disease: the good, the bad and the ugly. Clin Exp Immunol. 2011; 166: 1-15.

4. Mason DR, Beck PL, Muruve DA. Nucleotide-binding oligomerization domain-like receptors and inflammasomes in the pathogenesis of non-microbial inflammation and diseases. J Innate Immun. 2012; 4: 16-30.

5. Volt H, Garcia JA, Doerrier C, Diaz-Casado ME, Guerra-Librero A, Lopez LC, et al. Same molecule but different expression: aging and sepsis trigger NLRP3 inflammasome activation, a target of melatonin. J Pineal Res. 2016; 60: 193-205.

6. Sharif H, Wang L, Wang WL, Magupalli VG, Andreeva L, Qiao Q, et al. Structural mechanism for NEK7-licensed activation of NLRP3 inflammasome. Nature. 2019; 570: 338-43.

7. He Y, Hara H, Nunez G. Mechanism and Regulation of NLRP3 Inflammasome Activation. Trends Biochem Sci. 2016; 41: 1012-21.

8. Yabal M, Calleja DJ, Simpson DS, Lawlor KE. Stressing out the mitochondria: Mechanistic insights into NLRP3 inflammasome activation. J Leukoc Biol. 2019; 105: 377-99.

9. Lu A, Magupalli VG, Ruan J, Yin Q, Atianand MK, Vos MR, et al. Unified polymerization mechanism for the assembly of ASC-dependent inflammasomes. Cell. 2014; 156: 1193-206.

10. Dick MS, Sborgi L, Ruhl S, Hiller S, Broz P. ASC filament formation serves as a signal amplification mechanism for inflammasomes. Nat Commun. 2016; 7: 11929.

11. Dinarello CA. Immunological and inflammatory functions of the interleukin-1 family. Annu Rev Immunol. 2009; 27: 519-50.

12. Fink SL, Cookson BT. Caspase-1-dependent pore formation during pyroptosis leads to osmotic lysis of infected host macrophages. Cell Microbiol. 2006; 8: 1812-25.

13. Munoz-Planillo $R$, Kuffa P, Martinez-Colon G, Smith BL, Rajendiran TM, Nunez G. $\mathrm{K}(+)$ efflux is the common trigger of NLRP3 inflammasome activation by bacterial toxins and particulate matter. Immunity. 2013; 38: 1142-53.

14. Abais JM, Xia M, Zhang Y, Boini KM, Li PL. Redox regulation of NLRP3 inflammasomes: ROS as trigger or effector? Antioxid Redox Signal. 2015; 22: 1111-29.

15. Toldo $S$, Abbate A. The NLRP3 inflammasome in acute myocardial infarction. Nat Rev Cardiol. 2018; 15: 203-14.

16. Yaribeygi H, Mohammadi MT, Rezaee R, Sahebkar A. Fenofibrate improves renal function by amelioration of NOX-4, IL-18, and p53 expression in an experimental model of diabetic nephropathy. J Cell Biochem. 2018; 119: 7458-69

17. Grebe A, Hoss F, Latz E. NLRP3 Inflammasome and the IL-1 Pathway in Atherosclerosis. Circ Res. 2018; 122: 1722-40.

18. Zhang H, Gong X, Ni S, Wang Y, Zhu L, Ji N. C1q/TNF-related protein-9 attenuates atherosclerosis through AMPK-NLRP3 inflammasome singling pathway. Int Immunopharmacol. 2019; 77: 105934.

19. Ference BA, Ginsberg HN, Graham I, Ray KK, Packard CJ, Bruckert E, et al. Low-density lipoproteins cause atherosclerotic cardiovascular disease. 1. Evidence from genetic, epidemiologic, and clinical studies. A consensus statement from the European Atherosclerosis Society Consensus Panel. Eur Heart J. 2017; 38: 2459-72.

20. Hoseini Z, Sepahvand F, Rashidi B, Sahebkar A, Masoudifar A, Mirzaei H. NLRP3 inflammasome: Its regulation and involvement in atherosclerosis. J Cell Physiol. 2018; 233: 2116-32.

21. Kim HY, Lee HJ, Chang YJ, Pichavant M, Shore SA, Fitzgerald KA, et al. Interleukin-17-producing innate lymphoid cells and the NLRP3 inflammasome facilitate obesity-associated airway hyperreactivity. Nat Med. 2014; 20: 54-61. 
22. Ising C, Venegas C, Zhang S, Scheiblich H, Schmidt SV, Vieira-Saecker A, et al. NLRP3 inflammasome activation drives tau pathology. Nature. 2019; 575: 669-73.

23. Wang S, Yuan $\mathrm{YH}$, Chen $\mathrm{NH}$, Wang HB. The mechanisms of NLRP3 inflammasome/pyroptosis activation and their role in Parkinson's disease. Int Immunopharmacol. 2019; 67: 458-64.

24. Kimura $\mathrm{H}$. The physiological role of hydrogen sulfide and beyond. Nitric Oxide. 2014; 41: 4-10.

25. Wang R. Hydrogen sulfide: the third gasotransmitter in biology and medicine. Antioxid Redox Signal. 2010; 12: 1061-4.

26. Bian JS, Olson KR, Zhu YC. Hydrogen Sulfide: Biogenesis, Physiology, and Pathology. Oxid Med Cell Longev. 2016; 2016: 6549625.

27. Wang R. Physiological implications of hydrogen sulfide: a whiff exploration that blossomed. Physiol Rev. 2012; 92: 791-896.

28. Wang H, Shi X, Qiu M, Lv S, Liu H. Hydrogen Sulfide Plays an Important Protective Role through Influencing Endoplasmic Reticulum Stress in Diseases. Int J Biol Sci. 2020; 16: 264-71.

29. Szabo C, Papapetropoulos A. International Union of Basic and Clinical Pharmacology. CII: Pharmacological Modulation of H2S Levels: H2S Donors and H2S Biosynthesis Inhibitors. Pharmacol Rev. 2017; 69: 497-564.

30. Yang G, Wu L, Jiang B, Yang W, Qi J, Cao K, et al. H2S as a physiologic vasorelaxant: hypertension in mice with deletion of cystathionine gamma-lyase. Science. 2008; 322: 587-90.

31. Sun Y, Huang Y, Zhang R, Chen Q, Chen J, Zong Y, et al. Hydrogen sulfide upregulates KATP channel expression in vascular smooth muscle cells of spontaneously hypertensive rats. J Mol Med (Berl). 2015; 93: 439-55.

32. Guo C, Liang F, Shah Masood W, Yan X. Hydrogen sulfide protected gastric epithelial cell from ischemia/reperfusion injury by Keap1 s-sulfhydration, MAPK dependent anti-apoptosis and NF-kappaB dependent anti-inflammation pathway. Eur J Pharmacol. 2014; 725: 70-8.

33. Du J, Huang $Y$, Yan $H$, Zhang $Q$, Zhao $M$, Zhu M, et al. Hydrogen sulfide suppresses oxidized low-density lipoprotein (ox-LDL)-stimulated monocyte chemoattractant protein 1 generation from macrophages via the nuclear factor kappaB (NF-kappaB) pathway. J Biol Chem. 2014; 289: 9741-53.

34. Zheng J, Zhao T, Yuan Y, Hu N, Tang X. Hydrogen sulfide (H2S) attenuates uranium-induced acute nephrotoxicity through oxidative stress and inflammatory response via Nrf2-NF-kappaB pathways. Chem Biol Interact. 2015; 242: 353-62.

35. Luo ZL, Ren JD, Huang Z, Wang T, Xiang K, Cheng L, et al. The Role of Exogenous Hydrogen Sulfide in Free Fatty Acids Induced Inflammation in Macrophages. Cell Physiol Biochem. 2017; 42: 1635-44.

36. Huang Z, Zhuang X, Xie C, Hu X, Dong X, Guo Y, et al. Exogenous Hydrogen Sulfide Attenuates High Glucose-Induced Cardiotoxicity by Inhibiting NLRP3 Inflammasome Activation by Suppressing TLR4/NF-kappaB Pathway in H9c2 Cells. Cell Physiol Biochem. 2016; 40: 1578-90.

37. Olas B. Hydrogen Sulfide as a "Double-Faced" Compound: One with Pro- and Antioxidant Effect. Adv Clin Chem. 2017; 78: 187-96.

38. Chen J, Wang WH, Wu FH, He EM, Liu X, Shangguan ZP, et al. Hydrogen sulfide enhances salt tolerance through nitric oxide-mediated maintenance of ion homeostasis in barley seedling roots. Sci Rep. 2015; 5: 12516.

39. Cheung NS, Peng ZF, Chen MJ, Moore PK, Whiteman M. Hydrogen sulfide induced neuronal death occurs via glutamate receptor and is associated with calpain activation and lysosomal rupture in mouse primary cortical neurons. Neuropharmacology. 2007; 53: 505-14.

40. Wang HG, Sun WL, Zhong PY, Wu DD, Wang J, Cai $\mathrm{H}$, et al. Effect of exogenous hydrogen sulfide on expression of NLRP3 inflamma-some in hepatocytes. Chinese Journal of Pathophysiology 2018, 34(1) : 118-122

41. Wu D, Zhong P, Wang J, Wang H. Exogenous hydrogen sulfide mitigates LPS + ATP-induced inflammation by inhibiting NLRP3 inflammasome activation and promoting autophagy in L02 cells. Mol Cell Biochem. 2019; 457: 145-56.

42. Wang H, Zhong P, Sun L. Exogenous hydrogen sulfide mitigates NLRP3 inflammasome-mediated inflammation through promoting autophagy via the AMPK-mTOR pathway. Biol Open. 2019; 8.

43. Ji T, Han Y, Yang W, Xu B, Sun M, Jiang S, et al. Endoplasmic reticulum stress and NLRP3 inflammasome: Crosstalk in cardiovascular and metabolic disorders. J Cell Physiol. 2019.

44. Mu J, Cheng F, Wang Q, Wang X, Zhu W, Ma C, et al. Sini powder ameliorates the inflammatory response in rats with stress-induced non-alcoholic fatty liver disease by inhibiting the nuclear factor kappa-B / pyrin domain-containing protein 3 pathway. J Tradit Chin Med. 2020; 40: 253-66.

45. Dinis-Oliveira RJ, Duarte JA, Sanchez-Navarro A, Remiao F, Bastos ML, Carvalho F. Paraquat poisonings: mechanisms of lung toxicity, clinical features, and treatment. Crit Rev Toxicol. 2008; 38: 13-71.

46. Costa MD, de Freitas ML, Dalmolin L, Oliveira LP, Fleck MA, Pagliarini P, et al. Diphenyl diselenide prevents hepatic alterations induced by paraquat in rats. Environ Toxicol Pharmacol. 2013; 36: 750-8.

47. Han J, Zhang Z, Yang S, Wang J, Yang X, Tan D. Betanin attenuates paraquat-induced liver toxicity through a mitochondrial pathway. Food Chem Toxicol. 2014; 70: 100-6.

48. Liu Z, Zhao H, Liu W, Li T, Wang Y, Zhao M. NLRP3 inflammasome activation is essential for paraquat-induced acute lung injury. Inflammation. 2015; 38: 433-44.

49. Liu Z, Sun M, Wang Y, Zhang L, Zhao H, Zhao M. Silymarin attenuated paraquat-induced cytotoxicity in macrophage by regulating Trx/TXNIP complex, inhibiting NLRP3 inflammasome activation and apoptosis. Toxicol In Vitro. 2018; 46: 265-72

50. Liu Z, Wang X, Li L, Wei G, Zhao M. Hydrogen Sulfide Protects against Paraquat-Induced Acute Liver Injury in Rats by Regulating Oxidative Stress, Mitochondrial Function, and Inflammation. Oxid Med Cell Longev. 2020; 2020: 6325378 .

51. Hoste EA, Bagshaw SM, Bellomo R, Cely CM, Colman R, Cruz DN, et al. Epidemiology of acute kidney injury in critically ill patients: the multinational AKI-EPI study. Intensive Care Med. 2015; 41: 1411-23.

52. Abderrazak A, Syrovets T, Couchie D, El Hadri K, Friguet B, Simmet T, et al. NLRP3 inflammasome: from a danger signal sensor to a regulatory node of oxidative stress and inflammatory diseases. Redox Biol. 2015; 4: 296-307.

53. Chen Y, Jin S, Teng X, Hu Z, Zhang Z, Qiu X, et al. Hydrogen Sulfide Attenuates LPS-Induced Acute Kidney Injury by Inhibiting Inflammation and Oxidative Stress. Oxid Med Cell Longev. 2018; 2018: 6717212.

54. Liu Y. Cellular and molecular mechanisms of renal fibrosis. Nat Rev Nephrol. 2011; 7: 684-96

55. Vilaysane A, Chun I, Seamone ME, Wang W, Chin R, Hirota S, et al. The NLRP3 inflammasome promotes renal inflammation and contributes to CKD. J Am Soc Nephrol. 2010; 21: 1732-44.

56. Anders HJ, Suarez-Alvarez B, Grigorescu M, Foresto-Neto O, Steiger S, Desai $\mathrm{J}$, et al. The macrophage phenotype and inflammasome component NLRP3 contributes to nephrocalcinosis-related chronic kidney disease independent from IL-1-mediated tissue injury. Kidney Int. 2018; 93: 656-69.

57. Zhou Y, Zhu X, Wang X, Peng Y, Du J, Yin H, et al. H2S alleviates renal injury and fibrosis in response to unilateral ureteral obstruction by regulating macrophage infiltration via inhibition of NLRP3 signaling. Exp Cell Res. 2020; 387: 111779 .

58. Furuya F, Ishii T, Tamura S, Takahashi K, Kobayashi H, Ichijo M, et al. The ligand-bound thyroid hormone receptor in macrophages ameliorates kidney injury via inhibition of nuclear factor-kappaB activities. Sci Rep. 2017; 7: 43960.

59. Vural A, Nabar NR, Hwang IY, Sohn S, Park C, Karlsson MCI, et al. Galphai2 Signaling Regulates Inflammasome Priming and Cytokine Production by Biasing Macrophage Phenotype Determination. J Immunol. 2019; 202: 1510-20.

60. Wang J. Preclinical and clinical research on inflammation after intracerebral hemorrhage. Prog Neurobiol. 2010; 92: 463-77.

61. Chen S, Yang Q, Chen G, Zhang JH. An update on inflammation in the acute phase of intracerebral hemorrhage. Transl Stroke Res. 2015; 6: 4-8.

62. Ma $\mathrm{Q}$, Chen $\mathrm{S}, \mathrm{Hu} \mathrm{Q}$, Feng $\mathrm{H}$, Zhang JH, Tang J. NLRP3 inflammasome contributes to inflammation after intracerebral hemorrhage. Ann Neurol. 2014; 75: 209-19.

63. Zhao H, Pan P, Yang Y, Ge H, Chen W, Qu J, et al. Endogenous hydrogen sulphide attenuates NLRP3 inflammasome-mediated neuroinflammation by suppressing the P2X7 receptor after intracerebral haemorrhage in rats. J Neuroinflammation. 2017:14:163.

64. Liu C, Du Q, Zhang X, Tang Z, Ji H, Li Y. Clematichinenoside Serves as a Neuroprotective Agent Against Ischemic Stroke: The Synergistic Action of ERK1/2 and cPKC Pathways. Front Cell Neurosci. 2015; 9: 517.

65. Xia CY, Zhang S, Gao Y, Wang ZZ, Chen NH. Selective modulation of microglia polarization to M2 phenotype for stroke treatment. Int Immunopharmacol. 2015; 25: 377-82.

66. Ji J, Xiang P, Li T, Lan L, Xu X, Lu G, et al. NOSH-NBP, a Novel Nitric Oxide and Hydrogen Sulfide- Releasing Hybrid, Attenuates Ischemic Stroke-Induced Neuroinflammatory Injury by Modulating Microglia Polarization. Front Cell Neurosci. 2017; 11: 154

67. Haversen L, Danielsson KN, Fogelstrand L, Wiklund O. Induction of proinflammatory cytokines by long-chain saturated fatty acids in human macrophages. Atherosclerosis. 2009; 202: 382-93.

68. Moon JS, Lee S, Park MA, Siempos II, Haslip M, Lee PJ, et al. UCP2-induced fatty acid synthase promotes NLRP3 inflammasome activation during sepsis. J Clin Invest. 2015; 125: 665-80.

69. Duewell P, Kono H, Rayner KJ, Sirois CM, Vladimer G, Bauernfeind FG, et al. NLRP3 inflammasomes are required for atherogenesis and activated by cholesterol crystals. Nature. 2010; 464: 1357-61.

70. Abderrazak A, Couchie D, Mahmood DF, Elhage R, Vindis C, Laffargue M, et al. Anti-inflammatory and antiatherogenic effects of the NLRP3 inflammasome inhibitor arglabin in ApoE2.Ki mice fed a high-fat diet. Circulation. 2015; 131: 1061-70.

71. Sheedy FJ, Grebe A, Rayner KJ, Kalantari P, Ramkhelawon B, Carpenter SB, et al. CD36 coordinates NLRP3 inflammasome activation by facilitating intracellular nucleation of soluble ligands into particulate ligands in sterile inflammation. Nat Immunol. 2013; 14: 812-20.

72. Wang Y, Yan X, Mi S, Li Z, Wang Y, Zhu H, et al. Naoxintong attenuates Ischaemia/reperfusion Injury through inhibiting NLRP3 inflammasome activation. J Cell Mol Med. 2017; 21: 4-12

73. Gan W, Ren J, Li T, Lv S, Li C, Liu Z, et al. The SGK1 inhibitor EMD638683, prevents Angiotensin II-induced cardiac inflammation and fibrosis by blocking NLRP3 inflammasome activation. Biochim Biophys Acta Mol Basis Dis. 2018; 1864: 1-10.

74. Vandanmagsar B, Youm YH, Ravussin A, Galgani JE, Stadler K, Mynatt RL, et al. The NLRP3 inflammasome instigates obesity-induced inflammation and insulin resistance. Nat Med. 2011; 17: 179-88.

75. Jourdan T, Godlewski G, Cinar R, Bertola A, Szanda G, Liu J, et al. Activation of the Nlrp3 inflammasome in infiltrating macrophages by endocannabinoids mediates beta cell loss in type 2 diabetes. Nat Med. 2013; 19: 1132-40. 
76. Lin Z, Altaf N, Li C, Chen M, Pan L, Wang D, et al. Hydrogen sulfide attenuates oxidative stress-induced NLRP3 inflammasome activation via S-sulfhydrating c-Jun at Cys269 in macrophages. Biochim Biophys Acta Mol Basis Dis. 2018; 1864: 2890-900.

77. Castelblanco M, Lugrin J, Ehirchiou D, Nasi S, Ishii I, So A, et al. Hydrogen sulfide inhibits NLRP3 inflammasome activation and reduces cytokine production both in vitro and in a mouse model of inflammation. J Biol Chem. 2018; 293: 2546-57.

78. Ives A, Nomura J, Martinon F, Roger T, LeRoy D, Miner JN, et al. Xanthine oxidoreductase regulates macrophage IL1beta secretion upon NLRP3 inflammasome activation. Nat Commun. 2015; 6: 6555.

79. Xu W, Wu W, Chen J, Guo R, Lin J, Liao X, et al. Exogenous hydrogen sulfide protects $\mathrm{H} 9 \mathrm{c} 2$ cardiac cells against high glucose-induced injury by inhibiting the activities of the 338 MAPK and ERK1/2 pathways. Int J Mol Med. 2013; 32: 917-25.

80. Luo B, Li B, Wang W, Liu X, Liu X, Xia Y, et al. Rosuvastatin alleviates diabetic cardiomyopathy by inhibiting NLRP3 inflammasome and MAPK pathways in a type 2 diabetes rat model. Cardiovasc Drugs Ther. 2014; 28 : 33-43.

81. Wei WB, Hu X, Zhuang XD, Liao LZ, Li WD. GYY4137, a novel hydrogen sulfide-releasing molecule, likely protects against high glucose-induced cytotoxicity by activation of the AMPK/mTOR signal pathway in H9c2 cells. Mol Cell Biochem. 2014; 389: 249-56.

82. Zhou X, Lu X. Hydrogen sulfide inhibits high-glucose-induced apoptosis in neonatal rat cardiomyocytes. Exp Biol Med (Maywood). 2013; 238: 370-4

83. Cai J, Boulton M. The pathogenesis of diabetic retinopathy: old concepts and new questions. Eye (Lond). 2002; 16: 242-60.

84. Abu El-Asrar AM, Midena E, Al-Shabrawey M, Mohammad G. New developments in the pathophysiology and management of diabetic retinopathy. J Diabetes Res. 2013; 2013: 424258.

85. Wang P, Chen F, Wang W, Zhang XD. Hydrogen Sulfide Attenuates High Glucose-Induced Human Retinal Pigment Epithelial Cell Inflammation by Inhibiting ROS Formation and NLRP3 Inflammasome Activation. Mediators Inflamm. 2019; 2019: 8908960.

86. Hu TX, Zhang NN, Ruan Y, Tan QY, Wang J. Hydrogen sulfide modulates high glucose-induced NLRP3 inflammasome activation in 3T3-L1 adipocytes. Exp Ther Med. 2020; 19: 771-6.

87. Forbes JM, Cooper ME. Mechanisms of diabetic complications. Physiol Rev. 2013; 93: 137-88.

88. Zheng Q, Pan L, Ji Y. H 2S protects against diabetes-accelerated atherosclerosis by preventing the activation of NLRP3 inflammasome. J Biomed Res. 2019; 34: 94-102.

89. Wang HG, Zhang YJ, Huangfu CS, Chen ML, Wang J, Wu DD, et al. Effect of exogenous hydrogen sulfide on lipophagy in mouse primary hepatocytes.Chinese Journal of Pathophysiology 2017, 33(10) : 1901-1906.

90. Li F, Wiegman C, Seiffert JM, Zhu J, Clarke C, Chang Y, et al. Effects of $\mathrm{N}$-acetylcysteine in ozone-induced chronic obstructive pulmonary disease model. PLoS One. 2013; 8: e80782.

91. Li F, Zhang $\mathrm{P}$, Zhang $\mathrm{M}$, Liang $\mathrm{L}$, Sun $\mathrm{X}, \mathrm{Li} \mathrm{M}$, et al. Hydrogen Sulfide Prevents and Partially Reverses Ozone-Induced Features of Lung Inflammation and Emphysema in Mice. Am J Respir Cell Mol Biol. 2016; 55: 72-81.

92. Bauer C, Duewell P, Mayer C, Lehr HA, Fitzgerald KA, Dauer M, et al. Colitis induced in mice with dextran sulfate sodium (DSS) is mediated by the NLRP3 inflammasome. Gut. 2010; 59: 1192-9.

93. Zhao H, Yan R, Zhou X, Ji F, Zhang B. Hydrogen sulfide improves colonic barrier integrity in DSS-induced inflammation in Caco-2 cells and mice. Int Immunopharmacol. 2016; 39: 121-7.

94. Chen X, Liu XS. Hydrogen sulfide from a NaHS source attenuates dextran sulfate sodium (DSS)-induced inflammation via inhibiting nuclear factor-kappaB. J Zhejiang Univ Sci B. 2016; 17: 209-17.

95. Balaha M, Kandeel S, Elwan W. Garlic oil inhibits dextran sodium sulfate-induced ulcerative colitis in rats. Life Sci. 2016; 146: 40-51.

96. Qin M, Long F, Wu W, Yang D, Huang M, Xiao C, et al. Hydrogen sulfide protects against DSS-induced colitis by inhibiting NLRP3 inflammasome. Free Radic Biol Med. 2019; 137: 99-109.

97. Xue H, Zhou S, Xiao L, Guo Q, Liu S, Wu Y. Hydrogen sulfide improves the endothelial dysfunction in renovascular hypertensive rats. Physiol Res. 2015; 64: 663-72.

98. Xiao L, Dong JH, Jin S, Xue HM, Guo Q, Teng X, et al. Hydrogen Sulfide Improves Endothelial Dysfunction via Downregulating BMP4/COX-2 Pathway in Rats with Hypertension. Oxid Med Cell Longev. 2016; 2016: 8128957.

99. Li J, Teng X, Jin S, Dong J, Guo Q, Tian D, et al. Hydrogen sulfide improves endothelial dysfunction by inhibiting the vicious cycle of NLRP3 inflammasome and oxidative stress in spontaneously hypertensive rats. J Hypertens. 2019; 37: 1633-43.

100. Zhi L, Ang AD, Zhang H, Moore PK, Bhatia M. Hydrogen sulfide induces the synthesis of proinflammatory cytokines in human monocyte cell line U937 via the ERK-NF-kappaB pathway. J Leukoc Biol. 2007; 81: 1322-32.

101. Basic A, Alizadehgharib S, Dahlen G, Dahlgren U. Hydrogen sulfide exposure induces NLRP3 inflammasome-dependent IL-1beta and IL-18 secretion in human mononuclear leukocytes in vitro. Clin Exp Dent Res. 2017; 3: 115-20.

102. Hu X, Chi Q, Liu Q, Wang D, Zhang Y, Li S. Atmospheric H2S triggers immune damage by activating the TLR-7/MyD88/NF-kappaB pathway and NLRP3 inflammasome in broiler thymus. Chemosphere. 2019; 237: 124427. 